Ensayo

(Essay)

\title{
MIGUEL ÁNGEL MORÓN, LA TAXONOMÍA DE LOS MELOLONTHIDAE (COLEOPTERA: SCARABAEOIDEA) DE MÉXICO Y LA DIFUSIÓN DE SU CONOCIMIENTO
}

\author{
MIGUEL ANGEL MORON, THE TAXONOMY OF MELOLONTHIDAE \\ (COLEOPTERA: SCARABAEOIDEA) OF MEXICO AND DISSEMINATION \\ OF THEIR KNOWLEDGE
}

\author{
GONZALO HALFFTER
}

\author{
Instituto de Ecología, A. C., Red de Ecoetología. Carretera antigua a Coatepec \# 531, Col. El Haya, 91070 , \\ Xalapa, Veracruz, México <gonzalo.halffter@inecol.mx> \\ Recibido: 10/02/2018; aceptado: 13/02/2018; publicado en línea: 16/03/2018 \\ Editor responsable: Pedro Reyes Castillo
}

\begin{abstract}
Halffter, G. (2018) Miguel Ángel Morón, La Taxonomía de los Melolonthidae (Coleoptera: Scarabaeoidea) de México y la difusión de su conocimiento. Acta Zoológica Mexicana (nueva serie) 34(1): $1-4$.
\end{abstract}

RESUMEN. Como un homenaje póstumo a la labor de Miguel Ángel Morón en la taxonomía de los Melolonthidae de México, se presenta un análisis de su obra a partir de dos de sus libros y una exposición entomológica que motivo un tercer libro.

Palabras clave: Melolonthidae, Phyllophaga, taxonomía.

Como parte del homenaje que se rinde a un gran entomólogo (1 de diciembre 2017) Miguel Ángel Morón† (Fig. 1), me propongo evaluar su aporte a la taxonomía de los Melolonthidae de México, así como la amplia difusión que ha dado a este conocimiento. Me basaré en el análisis de dos de sus libros, así como en el de una original exposición itinerante que ha sido motivo de un tercer libro.

En el tardío desarrollo de las Ciencias Biológicas en México a partir de los años 60's del siglo XX (antes tenemos unos cuantos individuos, algunos notables, pero en general bastantes aislados), la taxonomía va a tener una importancia relativa superior a la que tenía en los países en aquel entonces punteros en la investigación biológica.

¿A qué se debió esta importancia relativa? (insisto en el término relativo). Creo que a varios factores: 1) La taxonomía es una actividad comparativamente barata y entonces, mucho más que ahora, no había dinero para
Halffter. G. (2018) Miguel Angel Moron, The Taxonomy of Melolonthidae (Coleoptera: Scarabaeoidea) of Mexico and dissemination of their knowledge. Acta Zoológica Mexicana (nueva serie) 34(1): $1-4$.

ABSTRACT. As a posthumous tribute to the work of Miguel Ángel Morón in the taxonomy of the Melolonthidae of Mexico, an analysis of his work is presented from two of his books and an entomological exhibition that gives rise to a third book.

Key words: Melolonthidae, Phyllophaga, taxonomy.

la ciencia; 2) La extraordinaria diversidad biológica de México era y es un atractivo real; 3) El hecho de que la casi totalidad de los brillantes biólogos españoles que fueron los principales maestros de la primera generación formal de biólogos mexicanos, maestros cuya influencia seguiría por lo menos durante diez años más, eran taxónomos. Nos referimos a personajes que han tenido tanta influencia como Federico Bonet, Cándido Bolívar, Faustino Miranda, Enrique Rioja y otros más. Miguel Ángel Morón no pertenece a la primera generación, pero si a la que se forma inmediatamente después bajo las mismas influencias.

Es interesante que varios de los investigadores que se iniciaron entonces, se dedican a los Scarabaeoidea. No es casual. Es producto del estímulo y retroalimentación que aparece cuando surge una "escuela" que establece vínculos y relaciones intelectuales. Nada es más frustrante para 


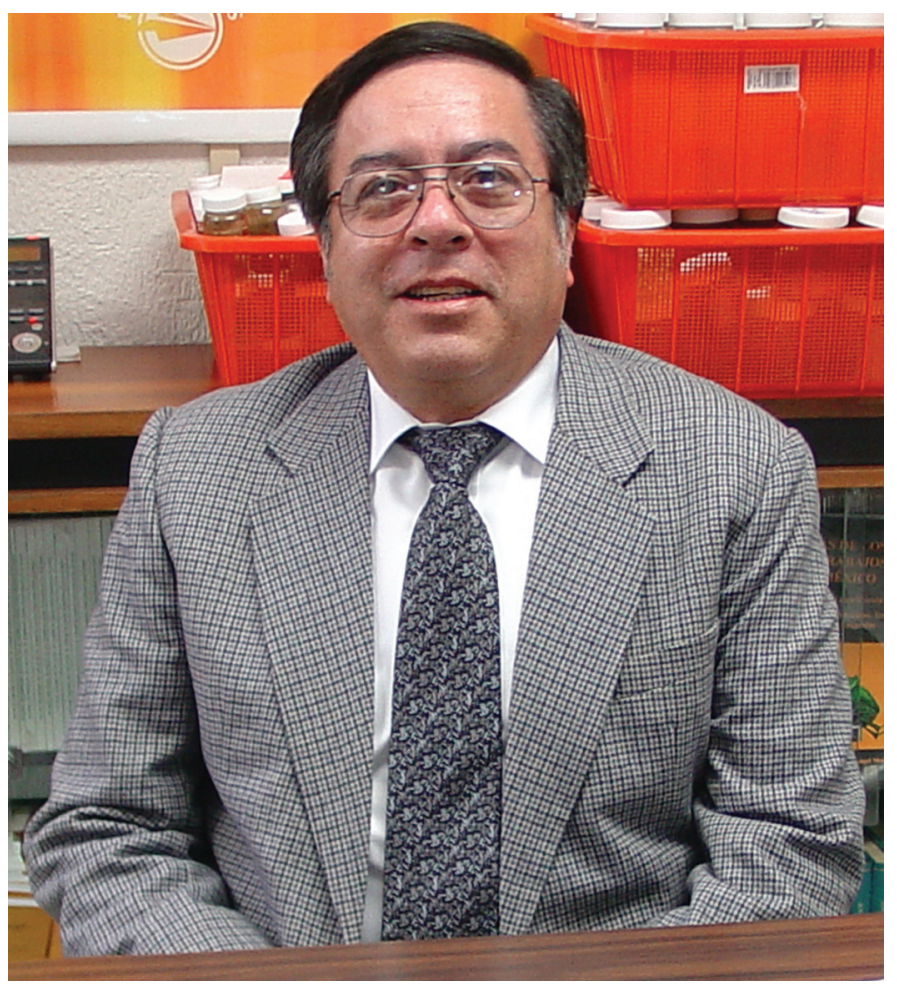

Figura 1. Dr. Miguel Ángel Morón Ríos` (24 de octubre de 1952 -22 de agosto de 2017).

el investigador que trabajar en la indiferencia. Por el contrario, la sensación y la participación en un movimiento es en sí el mejor estímulo.

En una entrevista que le hicieron en ocasión del premio de la Academia de la Investigación Científica (Moncada, 1993), Miguel Ángel contaba su juvenil atracción hacia los escarabajos en dos vertientes: colección y estudio. También sus primeros contactos con Alfredo Barrera, con Pedro Reyes-Castillo y conmigo en el Museo de Historia Natural de la Ciudad de México. A través de más de 45 años, Miguel Ángel Morón siguió con pasión y perseverancia esta precoz afición. Su producción científica es impresionante: 282 artículos (de los cuales 153 son sobre taxonomía de Melolonthidae adultos o larvas), 13 libros y 90 capítulos de libros, 102 artículos de divulgación y ensayos, y más de 52 tesis dirigidas, de las cuales 8 son de Doctorado. Él solo o en coautoría ha publicado una subtribu nueva, 13 géneros y 281 especies todos ellos nuevos para la ciencia, la mayor parte (pero no exclusivamente) de la fauna mexicana de Melolonthidae.

De esta rica y basta producción voy a analizar dos libros que considero muy significativos. Además, se me ha pedido contar mis recuerdos e información sobre una exposición itinerante excepcional, que dió lugar a otro libro.

\section{LA MONOGRAFÍA DE PHYLLOPHAGA DE MÉXICO}

La Tesis Doctoral de Miguel Ángel Morón (1985) es la revisión del género Phyllophaga en México, publicada al año siguiente (Morón, 1986) como libro por el Instituto de Ecología, A. C., entonces en la Ciudad de México. Es, sin duda, la publicación más significativa de Morón y una de las monografías más relevantes hechas en México. Un libro de verdadera relevancia internacional.

Como mejor Tesis Doctoral en el área de Ciencias Naturales recibió el Premio Weizmann 1987, otorgado por la Academia Mexicana de Ciencias en 1988. La misma Academia refrendo este honor, al otorgarle el Premio de Investigación Científica 1992 en el área de Ciencias Naturales, la distinción más importante que puede recibir un joven investigador mexicano. En la entrevista que con este motivo publicó la revista de la Academia (Moncada, 1993) Miguel Ángel comentaba que era la primera vez que esta distinción se daba a un taxónomo, lo que le llenó de orgullo.

En la Monografía de Phyllophaga en México, Miguel Ángel revisa y ordena las 254 especies del género que viven en el país. Analiza y describe los grupos (37) y los sub-géneros (8) existentes en México. El análisis taxonómico - morfológico abarca tanto a imagos como a larvas. Para este trabajo Morón estudio 52124 ejemplares entre 1977 y 1986, material situado en 20 colecciones de distintos países.

Para determinar qué importancia tiene el género Phyllophaga en el conjunto de la obra de Miguel Ángel Morón, he analizado su curriculum. No esperaba lo que encontré: dedicados específica y únicamente a especies de Phyllophaga, Morón publicó 96 artículos, un libro, 10 capítulos de libros y 6 artículos de divulgación. Caray!!

\section{ATLAS DE LOS ESCARABAJOS DE MÉXICO}

La siguiente obra a la que me voy a referir es distinta en estructura e intención. Se trata del "Atlas de los escarabajos de México", publicado en dos volúmenes. Morón no es el único autor de este libro, pero si su eje central.

Esta obra, singular en México y con escasos equivalentes en otros países, merece un análisis. De entrada, el 
nombre "Atlas" no corresponde. Creo que sería más correcto hablar de "Fauna" de los escarabajos de México, pues se trata de un compendio de fauna en el término más general e internacional de la palabra. Es una relación de todos los géneros y entidades supra-genéricas de escarabajos Lamellicornia existentes en México, incluyendo todas las especies en los géneros con pocas y una representación, en los más ricos en especies. Quizá su único antecedente, pero muy distinto, es la Biología CentraliAmericana, publicada hacia los años 80's del siglo XIX.

El Atlas, no es un libro de divulgación, tampoco es una monografía exhaustiva como la de Phyllophaga. Es una Fauna destinada a todos los que quieren tener una primera aproximación científica para conocer los Lamellicornia de México, su clasificación y un esquema de su distribución.

En los dos volúmenes, Morón da importancia al sistema de clasificación supragenérico. Para los Melolonthidae usa el modelo introducido por Endrödi en 1966. La taxonomía supra-genérica de los Lamellicornia tiene una movida historia. En los últimos años vuelve a ser tema de actualidad por la aparición de nuevos métodos, entre ellos los análisis filético-moleculares que ofrecen la posibilidad de clasificaciones más evolutivas.

Sobre el sistema de clasificación de los Melolonthidae, Miguel Ángel Morón tenía ideas bien asentadas, que defendió con calor. Afortunadamente conocemos su opinión gracias a un brillante análisis preparado con Bert Kohlmann en el 2003.

\section{EXPOSICIÓN Y LIBRO: "ESCARABAJOS: 200 MILLONES DE AÑOS DE EVOLUCIÓN"}

“Escarabajos: 200 Millones de Años de Evolución”, constituye una contribución notable y excepcional a la difusión del conocimiento. Exposición y libro forman un todo, por lo que hay que analizarlos como tal.

El proyecto se concibe en el Museo de Historia Natural de la Ciudad de México- Instituto de Ecología, A.C. (en aquellos años, hasta 1988 el Instituto estaba en los anexos del Museo). El proyecto, que se realiza en 1983, se propone integrar: 1) una exposición itinerante, 2) un ciclo de conferencias y 3 ) un libro que ampliara y detallara la exposición. El planteamiento museográfico estuvo a cargo de Yani Herreman; la dirección científica fue de Miguel Ángel Morón; Pedro Reyes Castillo dirigía el Museo e Instituto. El financiamiento lo otorgo la Dirección General Adjunta de Desarrollo Científico de Conacyt.
El emblema de la exposición y una de sus realizaciones más vistosas, fue una escultura de Dynastes hyllus (Fig. 2), un gran escarabajo casi exclusivo de México, que figuró en los carteles promocionales, en las invitaciones, y en las portadas del libro. El modelo de escarabajo tenía 92 centímetros de longitud y un peso de 34 kilogramos. Fue realizado por el escultor Pedro Dávalos, experto en la reproducción de arte precolombino y colonial, empleando fibra de vidrio con un acabado de resina acrílica pintada de tal forma que imitaba los colores naturales y el brillo ceroso pulido del insecto.

La parte principal de la exhibición estaba formada por 9 módulos construidos sobre bastidores de madera, con 41 bloques serigrafiados de textos, así como ampliaciones fotográficas a color o en blanco y negro. En cinco de estos módulos se ubicaron vitrinas con 570 ejemplares de escarabajos y en tres de los módulos se situaron seis dioramas, con escenas de la vida de seis especies de escarabajos. Estos dioramas fueron construidos con espuma de poliuretano. Para finalizar, se ubicaron algunos escarabajos de gran tamaño y muy llamativos y se agregaron 10 modelos elaborados con masilla de pan y pintados con óleo y laca para ilustrar un nido de Phanaeus, una pareja de Canthon rodando y el desarrollo de un Megasoma.

Después de ocho meses de preparación, la exposición fue inaugurada el 26 de noviembre de 1983 en el vestíbulo del Museo de Historia Natural de la Ciudad de México, en donde permaneció por dos meses. A continuación, vino el itinerario nacional. Gracias a los sencillos mecanismos dispuestos para plegar la colección, en un camión pequeño ocupando un volumen inferior a los 8 metros cúbicos,

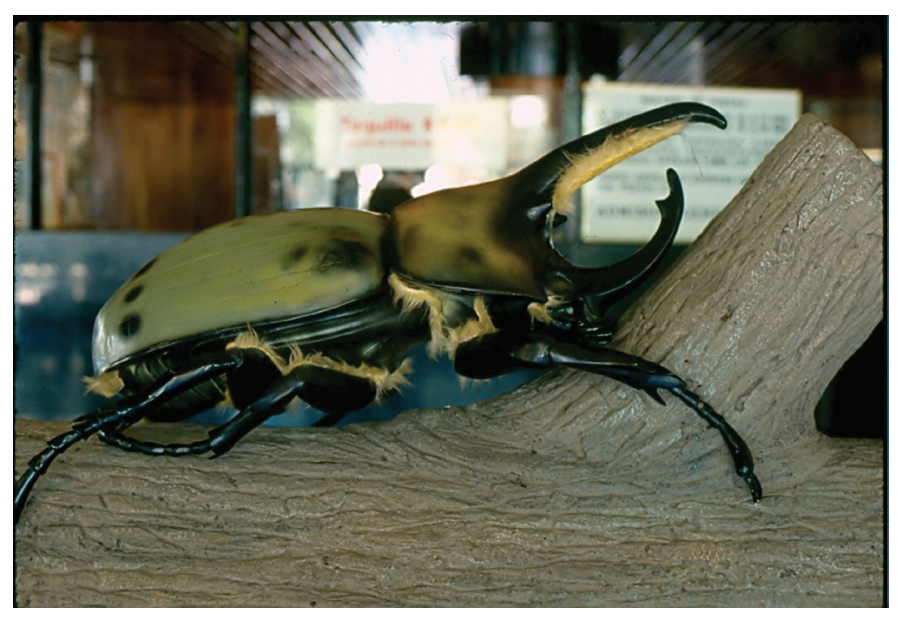

Figura 2. Modelo de Dynastes hyllus en la "Expo Sevilla, 1992". Foto G. Halffter. 
recorrió cerca de 15 mil kilómetros, para ser exhibida en 20 ciudades de la provincia mexicana y 10 instituciones del área metropolitana de la Ciudad de México. Según notas de Miguel Ángel la exposición fue vista por un mínimo de 5 millones de personas.

Entre 1983 y 1988 se dictaron 8 conferencias plenarias y más de 10 presentaciones para apoyar y divulgar los contenidos de la exposición itinerante. El libro que sintetiza está exposición, el atractivo y bien presentado "Escarabajos, 200 Millones de Años de Evolución" aparece en 1984. Ampliado y renovado, es reeditado en 2004. Agotada también la 2da. Edición, la versión electrónica, realizada por Cesar Rojas, aparece en el 2014.

Al principio de la década de los años 90's se inicia uno de los programas internacionales que más repercusión han tenido en la ciencia latinoamericana y española, el programa CYTED: Programa de Ciencia y Tecnología para el Desarrollo, que España, Portugal y los países de Iberoamérica impulsaron como en un compromiso de fraternidad. CYTED es el componente científico y tecnológico de la Cumbre de Jefes de Estado y de Gobierno de los países iberoamericanos.

El entonces Secretario Ejecutivo de CYTED (Dr. Jesús Sebastián) visitó el Museo de Historia Natural de México y quedo encantado con el Dynastes que teníamos en exhibición. Tan es así, que lo solicitó para la gran "Expo de Sevilla" con la que en el año de 1992 se conmemoraba los 500 años del Encuentro de Dos Mundos: España y América. El escarabajo quedó en el sitio más vistoso y llamativo del pabellón de CYTED, en el hall central de la Plaza de América en la "Expo Sevilla 1992". Permaneció en la feria durante todo el tiempo que ésta continuó.

En 1993, como Director General del Instituto de Ecología (ya en Xalapa) y como Coordinador Internacional del Subprograma Diversidad Biológica de CYTED, pude buscar los contactos y reunir los medios para que la presencia del Dynastes en la "Expo Sevilla" fuera el principio para una nueva itinerancia de toda la exposición, esta vez en España. Tanto Conacyt como CYTED pusieron fondos importantes para una renovación: cambio profundo y reparación del $60 \%$ del material. En consonancia con su incorporación al Programa de Diversidad Biológica de CYTED, se completó con una amplia muestra de la diversidad de los escarabajos: 150 ejemplares especialmente llamativos. Todo ello fue desarrollado por Miguel Ángel Morón.

Se estableció un acuerdo entre el Museo Nacional de Ciencias Naturales de Madrid y el Instituto de Ecología. El Museo de Madrid recibiría y expondría la colección y después la haría circular por España, para al terminar la itinerancia devolverla a México. La inauguración tuvo lugar el 20 de abril de 1993, en el Museo de Madrid. Hablaron el Dr. Fermín Martín Piera, muy distinguido entomólogo especialista en escarabajos y curiosamente autor del tomo de escarabajos de la Fauna Ibérica, o sea el equivalente español al Atlas que había hecho Miguel Ángel Morón en México. El segundo orador fue el propio Miguel Ángel Morón.

La exposición estuvo en Madrid hasta noviembre 1993. A partir de esa fecha se inicia una segunda itinerancia, esta vez por tierras españolas.

La exposición fue al Centro Cultural de Toledo, a Tolosa (en el País Vasco), al Museo de Zoología de Barcelona, a Durango (otra vez en el País Vasco), a Pamplona y cerraba diciembre 1995 en Sevilla. Son casi tres años de recorrer tierras españolas.

Según información, que estoy confirmando, actualmente se encuentra parcialmente expuesta en el Museo de Madrid.

AGRADECIMIENTOS. Por el apoyo en la búsqueda de información, al Biólogo Cesar Rojas (ayudante personal de Miguel Ángel Morón), a la Doctora Sara Larisa Gasperín (alumna de Morón) y al Biólogo Fernando Escobar. Así mismo al Doctor Jorge M. Lobo del Museo de Madrid.

\section{LITERATURA CITADA}

Kohlmann, B. \& Morón, M. A. (2003) Análisis histórico de la clasificación de los Coleoptera Scarabaeoidea o Lamellicornia. Acta Zoológica Mexicana (n.s.), 90, 175-280.

Moncada, G. (1993) Encuentro con Miguel Ángel Morón Ríos. Academia, 14, 37-41.

Morón, M. A. (1984) Escarabajos. 200 Millones de Años de Evolución. Instituto de Ecología, A. C. - Museo de Historia Natural de la Ciudad de México. $131 \mathrm{p}$.

Morón, M. A. (1986) El género Phyllophaga en México. Morfología, Distribución y Sistemática Supra Especifica (Insecta: Coleoptera). Instituto de Ecología, A. C. México, D. F. 341 p.

Morón, M. A. (editor). (2003) Atlas de los Escarabajos de México. Vol. II, Familias Scarabaeidae, Trogidae, Passalidae y Lucanidae. 12 láms., 202 p. Argonia editio, Barcelona.

Morón, M. A. (2004) Escarabajos. 200 Millones de Años de Evolución. 2da. Edición. 204 págs. Instituto de Ecología, A. C. Xalapa, Veracruz y Sociedad Entomológica Aragonesa, Zaragoza, España.

Morón, M. A., Ratcliffe, B. C. \& Deloya, C. (1997) Atlas de los Escarabajos de México, Coleoptera: Lamellicornia. Vol. I, Familia Melolonthidae. 280 p., 32 láms., CONABIO y Sociedad Mexicana de Entomología, A. C. 